\title{
STED nanoscopy combined with optical tweezers reveals protein dynamics on densely covered DNA
}

\author{
Iddo Heller ${ }^{1,2}$, Gerrit Sitters ${ }^{1,2}$, Onno D Broekmans ${ }^{1,2}$, Géraldine Farge ${ }^{1,2}$, Carolin Menges ${ }^{3}$, Wolfgang Wende ${ }^{3}$, \\ Stefan W Hell ${ }^{4}$, Erwin J G Peterman ${ }^{1,2}$ \& Gijs J L Wuite ${ }^{1,2}$
}

\begin{abstract}
Dense coverage of DNA by proteins is a ubiquitous feature of cellular processes such as DNA organization, replication and repair. We present a single-molecule approach capable of visualizing individual DNA-binding proteins on densely covered DNA and in the presence of high protein concentrations. Our approach combines optical tweezers with multicolor confocal and stimulated emission depletion (STED) fluorescence microscopy. Proteins on DNA are visualized at a resolution of $50 \mathrm{~nm}$, a sixfold resolution improvement over that of confocal microscopy. High temporal resolution $(<50 \mathrm{~ms}$ ) is ensured by fast one-dimensional beam scanning. Individual trajectories of proteins translocating on DNA can thus be distinguished and tracked with high precision. We demonstrate our multimodal approach by visualizing the assembly of dense nucleoprotein filaments with unprecedented spatial resolution in real time. Experimental access to the force-dependent kinetics and motility of DNA-associating proteins at biologically relevant protein densities is essential for linking idealized in vitro experiments with the in vivo situation.
\end{abstract}

Single-molecule studies have greatly enhanced our understanding of life at the molecular scale ${ }^{1,2}$. However, to obtain a high enough sensitivity and resolution for single-molecule studies, restrictive experimental conditions such as low protein density usually need to be imposed. Great challenges lie in overcoming these restrictions and performing experiments that reveal life at the single-molecule level in conditions similar to those in living cells.

Force spectroscopy ${ }^{3}$ and fluorescence microscopy $y^{2,4}$ provide access to global mechanical information and local structural information ${ }^{5}$, respectively, of single DNA-protein complexes ${ }^{6}$. The combination of wide-field fluorescence microscopy and optical tweezers enables localization of labeled proteins on optically stretched DNA with sub-10-nm precision ${ }^{5,7,8}$. Confocal fluorescence microscopy has also been integrated with optical tweezers, which allows analysis of fluctuations in fluorescence intensity within a fixed confocal volume ${ }^{9-11}$. Yet confocal imaging of optically trapped DNA remains unexploited despite its potential to resolve local binding, activity and motility of biomolecules on DNA at high concentrations of labeled protein in solution.

By providing nanoscale images from dense molecular environments, super-resolution fluorescence microscopy has prepared the foundation for carrying out single-molecule experiments at higher molecular density ${ }^{12}$. STED ${ }^{13}$ confocal nanoscopy stands out because it is a photon-efficient super-resolution technique in the sense that it requires many fewer photons per unit of time to probe molecular information than its spatially stochastic counterparts ${ }^{12}$. Thus, by facilitating fast imaging, STED nanoscopy should allow direct observation of protein dynamics on DNA. Additionally, great progress has been made in the last few years to neutralize elevated photobleaching induced by the STED beam, including the development of triplet relaxation approaches ${ }^{14,15}$ and the use of reductive-oxidative system (ROXS) buffers ${ }^{16}$.

Here we present a multimodal approach capable of elucidating the dynamics and mechanochemistry of complex cellular processes at high protein concentrations in solution on densely covered DNA. To this end, we designed an instrument that combines (i) optical tweezers, to control the conformation and tension on the DNA; (ii) beam-scanning confocal fluorescence microscopy, to image protein dynamics on DNA in a background of fluorescently labeled proteins; and (iii) one-dimensional (1D) STED nanoscopy, to distinguish individual labeled proteins and protein filaments at high protein density on DNA.

\section{RESULTS}

\section{Experimental setup}

Our experimental assay is based on a bacteriophage $\lambda$ DNA molecule tethered between two optically trapped microspheres ${ }^{17}$ (Fig. 1a). For optical trapping, two orthogonally polarized laser beams are focused by a 1.27-numerical aperture (NA) waterimmersion objective (Fig. 1b and Online Methods). Force is measured on both microspheres ${ }^{18}$, which allows enhancement of the force resolution through differential force detection ${ }^{19}$. For fluorescence microscopy, a pulsed laser system is used that provides both the STED and excitation pulses. A binary phase plate ${ }^{20}$, inserted in the path of the STED beam, enables 1D STED ${ }^{13}$.

\footnotetext{
${ }^{1}$ Department of Physics and Astronomy, Vrije Universiteit (VU University) Amsterdam, Amsterdam, The Netherlands. ${ }^{2}$ LaserLaB Amsterdam, VU University Amsterdam, Amsterdam, The Netherlands. ${ }^{3}$ Institute of Biochemistry, Justus Liebig University Giessen, Giessen, Germany. ${ }^{4}$ Department of NanoBiophotonics, Max Planck Institute for Biophysical Chemistry, Göttingen, Germany. Correspondence should be addressed to G.J.L.W. (g.j.l.wuite@vu.nl).

RECEIVED 17 OCTOBER 2012; ACCEPTED 1 JULY 2013; PUBLISHED ONLINE 11 AUGUST 2013; DOI:10.1038/NMETH.2599
} 
Figure 1 | Experimental layout. (a) Schematic of the measurement assay showing two optically trapped microspheres tethered by DNA. An excitation (EXC) beam (and superimposed STED beam) that is scanned over the DNA is shown in green. (b) Experimental setup showing beam paths and crucial components. For optical trapping (1,064 nm, dark red), two orthogonally polarized optical traps are independently steered using tip-tilt mirrors (M1 and M2), and microsphere displacements are measured on two position-sensitive detectors (PSDs) using back-focal-plane interferometry. The microsphere-to-microsphere distance is obtained from LED-illuminated complementary metal-oxide semiconductor (CMOS) camera images ( $875 \mathrm{~nm}$, purple). For fluorescence, one laser system supplies STED (745-nm, violet) and excitation beams (640 nm, $543 \mathrm{~nm}$ and $467 \mathrm{~nm}$, respectively red, green and blue, filtered from a supercontinuum spectrum (SC) using an acousto-optical tunable filter (A0TF)). These beams are fiber-coupled and delivered to the confocal tip-tilt piezo mirror scanner (M3) after being combined by dichroics (yellow). The descanned fluorescence signal (dashed lines) is collected on fiber-coupled avalanche photodiodes (APDs), and descanned excitation light can optionally be detected using a photomultiplier tube (PMT) by placing a pellicle beam splitter (BS, orange) in the common path. A STED stripe is formed by a binary phase plate (PP), and the excitation beams can optionally be circularly polarized using a $\lambda / 4$ retarder (QWP). Black and red stars indicate planes conjugate to the objective and condenser back focal plane, respectively. CL, condenser lens; D1-D11, dichroic mirrors; OL, water-immersion objective lens; T1-T3, telescopes.
$2 \mathrm{D}$ and $3 \mathrm{D}$ STED are also possible using alternative schemes (Online Methods). Optical manipulation can force DNA into a $1 \mathrm{D}$ configuration such that confocal beam scanning is required in only one dimension; the scan rate is limited by a tip-tilt piezo stage to $200 \mathrm{~Hz}$. We used relatively large (3.2- $\mu \mathrm{m}$-diameter) microspheres to spatially separate the fluorescent labels on the DNA-protein complexes from the trapping beams. This separation prevents photobleaching due to the simultaneous presence of fluorescence excitation and trapping laser beams ${ }^{9,21}$, and it simplifies instrument design and operation compared to the previously reported method of temporal separation ${ }^{11}$.
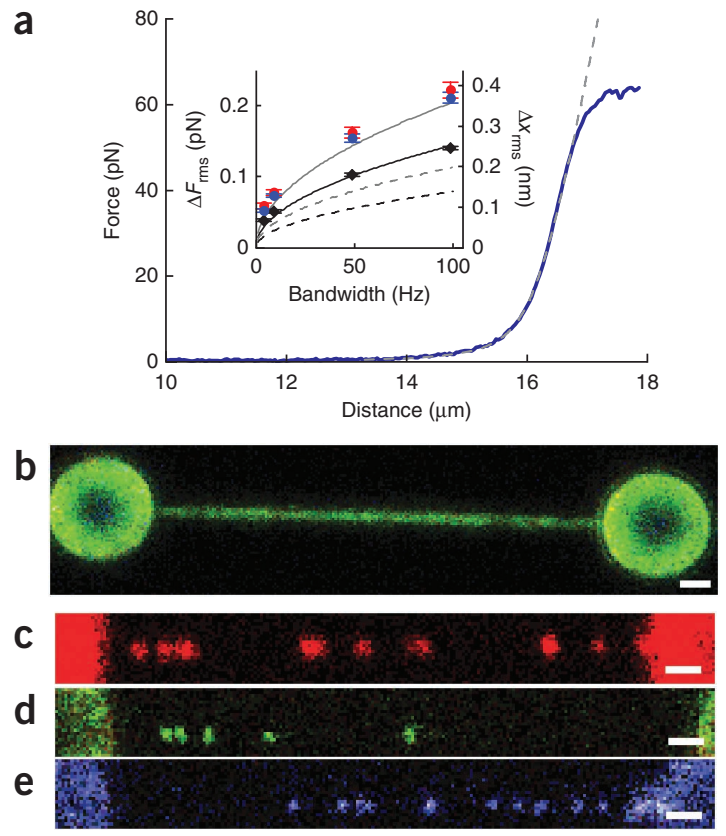

f

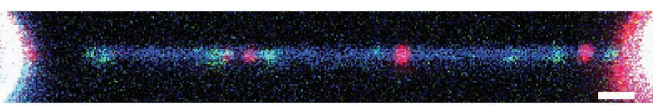

g

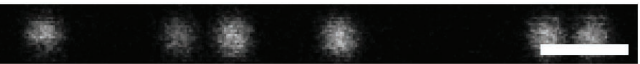

h

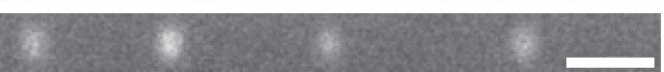

\section{Force spectroscopy with optical tweezers}

DNA-dumbbell constructs were created in situ ${ }^{22}$ and characterized by force-distance analysis (Fig. 2a). To test the performance of force spectroscopy with large microspheres, we quantified the resolution of our optical tweezers while stretching $\lambda$ DNA. The force and position fluctuations, respectively $\Delta F_{\mathrm{rms}}$ and $\Delta x_{\mathrm{rms}}$, were determined for the individual microspheres in each trap as well as for differential detection ${ }^{19}$ (Fig. 2a). The measured resolution, on the order of $0.1 \mathrm{pN}$ or $0.2 \mathrm{~nm}$, was in good agreement with the ab initio-calculated resolution limit set by thermal fluctuation of the dumbbell (Fig. 2a and Online Methods). For comparison, the resolution limit for widely used $900-\mathrm{nm}$-diameter microspheres was merely about twofold better.

\section{Multicolor confocal imaging and background rejection}

To demonstrate the confocal imaging capabilities of our instrument and to confirm single-fluorophore sensitivity, we imaged a range of fluorescent dyes on DNA (Fig. 2b-f). The instrument could reliably detect single fluorophores of Atto $647 \mathrm{~N}$,

Figure 2 | Force spectroscopy and confocal fluorescence microscopy. (a) Experimental force-distance curve of $\lambda$ DNA in PBS. The dashed curve shows the extendible wormlike-chain model calculated using a persistence length of $55 \mathrm{~nm}$, a contour length of $16.5 \mu \mathrm{m}$ and a stretching modulus of $1,350 \mathrm{pN}$. DNA overstretching occurs near $65 \mathrm{pN}$. Inset, measured (symbols, s.d.) and calculated (curves) force and position noise ( $\Delta F$ and $\Delta x$, respectively) as a function of measurement bandwidth (average trap stiffness $=0.57 \mathrm{pN} / \mathrm{nm} ; F=25 \mathrm{pN}$ ). Red, blue and gray, noise for singlebead detection; black, noise for differential detection; solid curves, 3.2- $\mu \mathrm{m}$-diameter microspheres; dashed curves, 0.9- $\mu \mathrm{m}$-diameter microspheres. (b) Confocal microscopy image of Sytox Orange-labeled $\lambda$ DNA between two 3.2- $\mu \mathrm{m}$ microspheres (excitation (exc.), $543 \mathrm{~nm}$ ). (c-e) Confocal microscopy images of individual EcoRV-Atto 647N (c; exc., $640 \mathrm{~nm}$ ), Sytox Orange dyes (d; exc., $543 \mathrm{~nm}$ ) and EGFP-labeled proteins (e; exc., $467 \mathrm{~nm}$ ). (f) Simultaneous multicolor imaging of Sytox Orange, Sytox Blue and BsoBI-Atto 647N. (g,h) Confocal microscopy images of individual BsoBI-Atto $647 \mathrm{~N}$ restriction enzymes bound specifically to optically stretched $\lambda$ DNA in the absence $(\mathbf{g})$ and presence $(\mathbf{h})$ of $100 \mathrm{nM}$ of free Atto 647N NHS-ester (the local concentration in the flow cell is estimated to be lower owing to adsorption, approximately $40 \mathrm{nM}$ ). Scale bars, $1 \mu \mathrm{m}$. The typical frame rate for a $25 \mu \mathrm{m} \times 2 \mu \mathrm{m}$ field of view using $100-\mu$ s dwell time per $75-\mathrm{nm}$ pixel is $1 \mathrm{~Hz}$. 


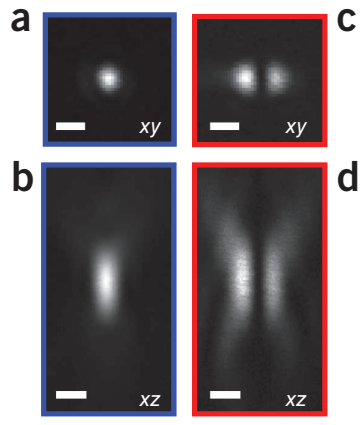

Figure 3 | Characterization of STED nanoscopy of proteins on optically stretched DNA.

(a-d) Focal-intensity distributions of the 640-nm excitation $(\mathbf{a}, \mathbf{b})$ and 745-nm STED beam $(\mathbf{c}, \mathbf{d})$ imaged in the $x y(\mathbf{a}, \mathbf{c})$ and $x z(\mathbf{b}, \mathbf{d})$ planes. Scale bars, $500 \mathrm{~nm}$. (e,f) Confocal microscopy (e) and subsequent STED image (f; power $P_{\text {STED }}=6 \mathrm{~mW}$ ) of EcoRV-Atto $647 \mathrm{~N}$ on $\lambda$ DNA. Scale bars, $1 \mu \mathrm{m}$. (The ellipticity of the intensity distributions in $\mathbf{e}$ is likely due to linear polarization of the excitation light, lateral DNA fluctuations ${ }^{5}$ or aberrations associated with residual tilt of the coverglass with respect to the water-immersion lens.) $(\mathbf{g}, \mathbf{h})$ Scaling of effective resolution with $P_{\text {STED }}(\mathbf{g} ; F=4 \mathrm{pN})$ and with force $\left(\mathbf{h} ; P_{\text {STED }}=4 \mathrm{~mW}\right)$, showing the average FWHM $(\langle n\rangle=9.7)$ of Gaussian fits to intensity profiles obtained from BsoBI-Atto 647N and EcoRV-Atto 647N on DNA (vertical error bars, s.e.m.; horizontal error bars (h only), s.e. $=0.2 \mathrm{pN}$ ). Solid lines, fits to data; dashed line, amplitude of longitudinal DNA fluctuations (Online Methods). The insets show projected intensity profiles of Atto $647 \mathrm{~N}$-labeled enzymes on DNA for the DNA section indicated by the dashed lines in $\mathbf{e}\left(\mathbf{g}\right.$, blue) and $\mathbf{f}\left(\mathbf{g}\right.$, red) and for $P_{\text {STED }}=4 \mathrm{~mW}$ at $0.4-\mathrm{pN}$ (h, black) and 30-pN (h, red) tension. (i) Average number of photons detected from individual fluorophores before photobleaching (s.e.m., $\langle n\rangle=43$ ), obtained from exponential fits of photon-count distributions as in the inset, for Tris (gray) and reductive-oxidative system (red) buffers at $P_{\text {STED }}=0 \mathrm{~mW}, 6 \mathrm{~mW}$ and $16 \mathrm{~mW}$.

Sytox Orange and EGFP (Fig. 2c-e and Supplementary Fig. 1). Simultaneous multicolor imaging enables more complex, multicomponent biomolecular studies (Fig. 2f). Notably, no elevated photobleaching rate was observed near the optically trapped microspheres during confocal imaging (Fig. 2b-f).

To qualitatively test background rejection in our confocal imaging layout, we imaged Atto $647 \mathrm{~N}$-labeled proteins on DNA in the absence (Fig. 2g) or presence (Fig. 2h) of free dye in solution ( $40 \mathrm{nM}$ Atto $647 \mathrm{~N}$ NHS-ester). With a 1-ms integration time, we estimated the upper limit of background concentration for which we could obtain single-molecule sensitivity to be about $100 \mathrm{nM}$, which is approximately 1-2 orders of magnitude higher than the typical limit for wide-field imaging.

\section{STED nanoscopy of proteins on DNA}

We employed STED to perform subdiffraction imaging in optical tweezers. STED allows fast imaging on DNA at a rate that is ultimately limited by the scanning speed and the rate of fluorescence emission. For STED, we used a focal-intensity distribution that features a 1D central line of nearly zero intensity ${ }^{20}$ (Fig. 3a-d and Online Methods) rather than a central zero point of a doughnutshaped distribution ${ }^{23}$. By orienting the zero line perpendicular to the stretched DNA, we enhanced the spatial resolution along the length of the DNA only. This 1D-STED scheme renders 1D line scanning far less sensitive to lateral DNA fluctuations, misalignment or drift between the optical trapping and fluorescence imaging systems than does a doughnut.

A comparison of confocal and STED images of Atto $647 \mathrm{~N}-$ labeled restriction enzymes specifically bound to optically stretched DNA demonstrated the clear resolution enhancement along the DNA that STED provides (Fig. 3e-g). Notably, no significant additional DNA nicking was observed during STED imaging (Supplementary Note 1).

Precise control of tension on the DNA is important to achieve high-resolution imaging. Thermal fluctuations of suspended DNA will blur the images taken of DNA-bound proteins ${ }^{5}$. However, the amplitude of such fluctuations can be reduced by applying tension to the DNA using optical tweezers. We found that reducing the DNA tension from $30 \mathrm{pN}$ to $0.4 \mathrm{pN}$ completely abolished the ability of STED nanoscopy to resolve two DNA-bound proteins in close proximity (Fig. $3 \mathbf{h}$ ). We measured the full-width at halfmaximum (FWHM) of STED images of single Atto 647N-labeled proteins as a function of applied force, and the results revealed a loss of effective resolution at forces below $\sim 5 \mathrm{pN}$. Our data are well-described by a model (Fig. $3 \mathbf{h}$ ) that convolves the optical resolution with estimated longitudinal DNA fluctuations (Online Methods).

We further characterized the scaling of resolution with STED power $\left(P_{\text {STED }}\right)$ by imaging restriction enzymes on optically stretched DNA (Fig. 3g). At $P_{\text {STED }}=26 \mathrm{~mW}$, we obtained a FWHM resolution of $50 \pm 5 \mathrm{~nm}$ (s.e.m.) corresponding to a sixfold resolution enhancement over that of confocal imaging under the same conditions (given by the water-immersion lens and the aqueous medium). The scaling of resolution with $P_{\text {STED }}$ is well described by taking into account the blurring due to thermal fluctuation of the DNA (Fig. 3g, Online Methods and Supplementary Fig. 2). Most notably, the gain in resolution allows the imaging of DNAprotein complexes in optical tweezers at a resolution comparable to the persistence length of DNA.

To characterize photobleaching, we counted the number of photons collected from individual Atto 647N fluorophores on optically stretched DNA before bleaching (Fig. 3i). For confocal 
Figure 4 | Characterization of localization precision. (a,b) Kymographs of Atto $647 \mathrm{~N}$-labeled proteins on optically stretched DNA, imaged partially by confocal microscopy and partially by STED nanoscopy $\left(P_{\text {STED }}=\right.$ $6 \mathrm{~mW}$ ), for immobilized EcoRV-Atto 647N (a) and for diffusing TFAM-Atto $647 \mathrm{~N}$ (b). Scale bars, $200 \mathrm{~nm}$. Red lines track the centers of the photon distributions and were obtained by fitting 1D Gaussians to each pixel column. (c) Power-spectral density (PSD) for tracked positions of proteins on DNA. Black squares, PSD for a single diffusing TFAM-Atto $647 \mathrm{~N}$; dashed line, $1 / f^{2}$ behavior; circles, averaged PSDs for immobilized BsoBI-Atto $647 \mathrm{~N}$ for confocal imaging (open blue circles, $n=6$ ) and STED imaging (filled red circles, $n=10$ ). (d) Localization precision $\Delta x_{\text {rms }}$ as function of $P_{\text {STED }}(F=4 \mathrm{pN})$. Red symbols, localization precision as obtained from tracking immobilized proteins on DNA (s.e.m., $\left.\langle n\rangle=10.9\right)$; solid curve, ideal localization precision that neglects DNA fluctuations and is governed solely by photon-counting noise, pixelation noise and background noise; dashed curve, optimal ideal localization precision that assumes no loss in peak brightness of the fluorophores with increasing STED power (Online Methods).

imaging in Tris buffer, about 55,000 photons could be collected. For STED imaging, however, about one and two orders of magnitude fewer photons were collected for $P_{\text {STED }}=6 \mathrm{~mW}(87-\mathrm{nm}$ FWHM) and $P_{\text {STED }}=16 \mathrm{~mW}(64-\mathrm{nm}$ FWHM), respectively, a signal reduction that is comparable to that observed under two-photon excitation ${ }^{24}$. We note that in our case, some of the photobleaching may also be due to the fact that in our high-NA water-immersion system, the STED focal intensity was not perfectly 0 at the minimum: a deviation that starts having an effect at higher STED powers. Hence, further optimization of the STED focal beam, which in the water immersion case critically relies on the coverglass tilt with respect to the lens, may increase the photon yield further. Nevertheless, it is important to note that the 500-5,000 photons collected per fluorophore (at 64-nm FWHM to $87-\mathrm{nm}$ FWHM, respectively) are sufficient for imaging up to 50-500 frames, thereby allowing nanoscale observation of protein dynamics over extended periods of time (see below). By using antibleaching buffer ROXS (Online Methods and Supplementary Fig. 3), we could increase the number of collected photons about two- to threefold (i.e., to $~ 1,000$ photons at $64-\mathrm{nm}$ FWHM).

Next we explored the localization precision of proteins for confocal and STED imaging by acquiring kymographs of immobilized restriction enzymes on DNA (Fig. 4a). For comparison, we also imaged a protein that undergoes $1 \mathrm{D}$ diffusion on DNA (Fig. 4b). We tracked the position of these proteins and calculated the power-spectral density of fluctuations in the tracked position (Fig. 4c). Whereas a diffusing protein exhibited a power spectrum with $1 / f^{2}$ frequency $(f)$ dependence, which is characteristic for free diffusion, immobilized proteins exhibited a frequencyindependent power spectrum. The latter power spectra represent the experimental noise floor for particle tracking. Notably, 1D-STED imaging provided an enhanced localization precision (i.e., lower noise floor) compared to confocal imaging, enabling observation of protein translocation at higher spatial or temporal resolution.

To further quantify the localization precision, we plotted the r.m.s. fluctuations of the tracked position, $\Delta x_{\text {rms }}$, for immobilized enzymes as function of $P_{\text {STED }}$ (Fig. 4d). Indeed, a near-twofold enhancement of the localization precision of 1D STED over confocal microscopy was observed, saturating at $\sim 6 \mathrm{~nm}$ for high $P_{\text {STED. }}$. Analysis of the measured localization precision provides information on the stability and reliability of STED imaging on optically stretched DNA. The 'ideal' localization precision, neglecting DNA fluctuations, is governed solely by photon-counting noise, pixelation noise and background noise ${ }^{25}$ (Fig. 4d and Online Methods). Of note, the ideal localization precision was only $\sim 2 \mathrm{~nm}$ better than the measured localization precision. This illustrates that STED super-resolution imaging on optically manipulated DNA is highly reliable and stable. The additional experimental noise may include contributions from slow thermal fluctuations of the dumbbell construct, from drift and fluctuations between the separate optical trapping and confocal imaging systems, and from the line-to-line repeatability of the confocal beam scanner. Furthermore, the observed saturation of localization precision at high $P_{\text {STED }}$ implies suboptimal tracking, which can be due to a reduced maximum fluorescence emission rate from the nonzero intensity of the focal STED beam. Figure $4 \mathbf{d}$ also indicates the calculated optimal localization precision when there is no loss in peak brightness of the fluorophores with increasing STED power. In the optimal 1D-STED case, the photon-counting noise scales as $\sqrt{F W H M}$. Intriguingly, the enhanced photon yield by ROXS should be sufficient to obtain single-base-pair localization precision in this optimal case (i.e., by collecting 10,000 photons at $\left.P_{\text {STED }}=6 \mathrm{~mW}\right)$.

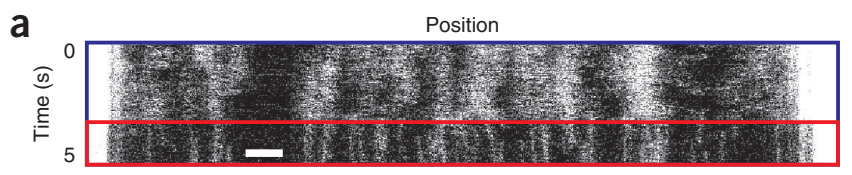

b

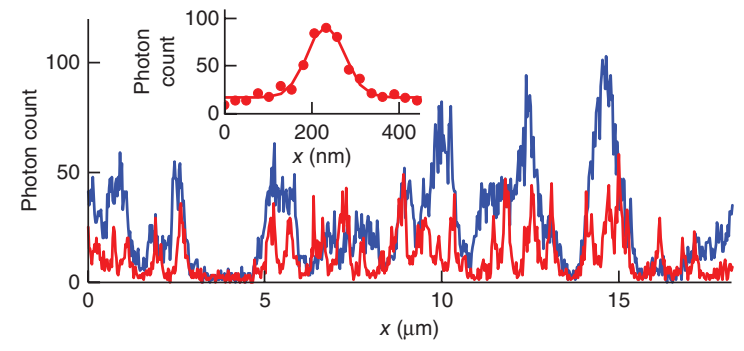

Figure 5 | STED nanoscopy of Sytox Red on optically stretched DNA. (a) Kymograph showing Sytox Red dyes binding to double-stranded $\lambda$ DNA. Scale bar, $1 \mu \mathrm{m}$. The top (blue) was acquired by confocal microscopy, whereas the bottom (red) was recorded using STED nanoscopy $\left(P_{\text {STED }}=6 \mathrm{~mW}\right)$. (b) Intensity profiles obtained from the confocal (blue) and STED (red) imaging sections of a. The inset shows the intensity profile of an individual Sytox Red dye bound to DNA. A Gaussian fit (line) yields a FWHM of $99 \mathrm{~nm}$. 
Figure 6 | TFAM binding and diffusion dynamics on optically stretched DNA. $(\mathbf{a}, \mathbf{b})$ Kymographs of TFAM-Atto $647 \mathrm{~N}$ dynamics on $\lambda$ DNA $(F=4 \mathrm{pN})$. Scale bars, $1 \mu \mathrm{m}$. In a, the DNA is moved into a channel with $50 \mathrm{nM}$ human TFAM-Atto 647N (estimated concentration is lower owing to adsorption: $\sim 5 \mathrm{nM}$ ) and imaged using confocal microscopy. In $\mathbf{b}$, the DNA is in reductiveoxidative system buffer, and at $t=\sim 66 \mathrm{~s}$, the STED beam is switched on at $6 \mathrm{~mW}$ (red borders; $\mathrm{FWHM}=75 \mathrm{~nm}$ ). Arrows, proteins that diffuse within diffraction-limited regions; asterisks, oligomers that break up again. Imaging in a and $\mathbf{b}$ was performed at $10 \mu$ s per $25-\mathrm{nm}$ pixel and a line rate of $90 \mathrm{~Hz}$; five-line averages are displayed. $(\mathbf{c}, \mathbf{d})$ Histograms of the intensity of TFAM-Atto $647 \mathrm{~N}$ on DNA normalized to the number of fluorophores, $I_{\text {norm }}($ c) and of the diffusion coefficient $D(\mathbf{d})$, obtained by both STED and confocal imaging. Blue bars, TFAM immediately after binding DNA $(n=42)$; red bars, the full data set $(n=143)$, including already-bound TFAM and TFAM filaments.

$D$ was calculated by fitting the mean-squared displacement (MSD) determined from individual traces (number of lines $=150 \pm 75$ (mean \pm s.d.), with a minimum of 50 lines) to MSD $=2 D t+$ offset. Gray curves, normalized distributions of $D$ for simulated diffusion data (Online Methods) with $D_{\text {ref }}=0.08 \mu \mathrm{m}^{2} / \mathrm{s}$ (dashed) and with a range of diffusion constants $D=D_{\text {ref }} / N_{\text {TFAM }}$, where $N_{\text {TFAM }}=1,2,3, \ldots, 12$ (solid) (Supplementary Note 2). (e) Kymograph of a TFAM-Atto $647 \mathrm{~N}$ oligomerization event (line rate $=122 \mathrm{~Hz}$; five-line average displayed). (f) MSD analysis of trajectories i-iii as indicated in e (s.d.; mean number of lines $=130$ ).

Finally, we demonstrated that dsDNA could also be imaged with subdiffraction resolution using STED ${ }^{26}$ (Fig. 5). In the kymograph, acquired sequentially by confocal and STED microscopy, binding of individual Sytox Red dyes to DNA could be observed (Fig. 5a). The intensity profiles showed a resolution enhancement by a factor of 3 over confocal microscopy resolution (Fig. 5b).

\section{Imaging protein dynamics at high protein density}

We further demonstrated our multimodal approach by studying the DNA-binding dynamics of the human mitochondrial transcription factor TFAM ${ }^{27,28}$ labeled with Atto $647 \mathrm{~N}$ (Fig. 6). In contrast to earlier wide-field microscopy recordings ${ }^{29}$, confocal microscopy allows real-time observation of individual TFAM-Atto $647 \mathrm{~N}$ binding to DNA in a solution containing up to $\sim 100 \mathrm{nM}$ TFAM-Atto $647 \mathrm{~N}$. Indeed, we observed binding followed by diffusion of TFAM-Atto $647 \mathrm{~N}$ on stretched DNA (Fig. 6a). The initial TFAM intensity distribution, measured immediately after binding, peaked at one fluorophore (Fig. 6c). This is consistent with previous findings that TFAM primarily binds DNA as mono$\operatorname{mer}^{29}$ (a labeling ratio below unity, however, prevents us from extensive interpretation of the fluorescence intensity).

In vivo, the abundance of TFAM in mitochondria is large enough to coat the entire mitochondrial genome and form filaments on DNA. We intended to mimic this regime in vitro by studying TFAM-coated DNA at high TFAM concentration. However, confocal microscopy cannot resolve individual protein trajectories at high protein densities (Fig. 6a). Using STED, in contrast, we observed many TFAM-Atto $647 \mathrm{~N}$ molecules diffusing on DNA (Fig. 6b). STED allowed observation of individual protein (filament) trajectories along the DNA at subdiffraction resolution. Bright regions that might be misinterpreted as stable filaments in confocal microscopy were identified using STED to consist of individual TFAM-Atto $647 \mathrm{~N}$ proteins or filaments that diffuse rapidly within diffraction-limited regions (Fig. 6b). In addition, STED nanoscopy over periods $>20$ s clearly revealed that TFAM molecules oligomerize after collision with other TFAM molecules, as reported before ${ }^{29}$. Although the imaging resolution in this experiment was insufficient to distinguish adjacent proteins within an oligomer ( $10-\mathrm{nm}$ separation), repeated imaging revealed whether proteins were in mere coincidental proximity or were in fact diffusing colocalized in an oligomer over an extended period of time. Notably, such oligomers can occasionally break up again (Fig. 6b). We used the maximum STED resolution to image TFAM on DNA at even higher density (Supplementary Fig. 4).

Furthermore, STED allows individual proteins to be tracked at high density with less ambiguity, providing statistics for larger particle numbers and allowing studies of diffusive behavior on crowded DNA. We performed mean-square-displacement (MSD) analysis of individual TFAM-Atto 647N trajectories on DNA in order to estimate the diffusion constants $D$ (Fig. 6d). Interestingly, we found a mean diffusion constant of $0.028 \pm 0.004 \mu \mathrm{m}^{2} / \mathrm{s}$ (s.e.m.; $n=143$ ), which is much slower than the diffusion constant measured previously for TFAM monomers that diffuse on very sparsely covered DNA ${ }^{29}: D_{\text {ref }} \approx 0.08 \mu \mathrm{m}^{2} / \mathrm{s}$. This finding suggests that at the high TFAM density used here, TFAM oligomerization occurs, which can reduce $D$. One such oligomerization event is shown in Figure 6e. Indeed, the slope of the MSD-versus-time curve is reduced by $\sim 50 \%$ after oligomerization (Fig. 6f), indicating an $\sim 50 \%$ reduction in $D$. The expected distribution of $D$ for a single population of TFAM monomers with $D=D_{\text {ref }}$ (Fig. 6d), calculated using simulated diffusion data (Online Methods), fails to describe the measured data. Instead, a simulation that assumes a 
mix of oligomers having different diffusion constants (Fig. 6d and Supplementary Note 2) is in good agreement with the measured diffusion data.

\section{DISCUSSION}

We introduced a multimodal approach that combines optical tweezers with confocal and STED microscopy. Confocal microscopy allows imaging of single DNA-bound proteins on optically stretched DNA at background concentrations up to two orders of magnitude higher than those of wide-field microscopy. A time resolution comparable to that of typical wide-field imaging is ensured by fast confocal line scanning along DNA that is linearized by optical tweezers. Our technique enables new experiments at biologically relevant protein concentrations wherein protein binding and dynamics can be spatially resolved in real time at the high time resolution of $50 \mathrm{~ms}$ (up to $5 \mathrm{~ms}$ ). Multicolor imaging enables more complex studies of multicomponent biomolecular systems.

Super-resolution imaging provides a sixfold resolution enhancement over confocal imaging, thereby granting unique access to more physiologically compatible conditions, in which DNA is densely coated with proteins. We demonstrated a spatial resolution of $50 \mathrm{~nm}$, which is directly comparable to biologically interesting length scales such as the persistence length of dsDNA, the length of eukaryotic Okazaki fragments and typical nucleosomenucleosome spacings in a beads-on-a-string configuration. Notably, the time resolution of confocal imaging is not compromised by STED. In fact, the time resolution of localization and tracking is enhanced owing to better localization accuracy. STED thus allows observation of rapid dynamics with subdiffraction resolution. As an alternative to STED, stochastic super-resolution techniques based on single-molecule on-off switching are optically less complex and can be readily integrated with wide-field fluorescence on optically stretched DNA ${ }^{30}$. These techniques, however, are currently slower than STED because larger numbers of detected photons are needed to localize the position of the molecules and to construct images. In STED microscopy, the fluorescence on-off switching occurs instantly and in a spatiotemporally controlled way, which facilitates the implementation of fast imaging.

Optical tweezers allow precise control of DNA location and tension, which is crucial for suppressing thermal fluctuations that can inhibit subdiffraction imaging. In addition, the 1D-STED scheme prevents problems associated with lateral DNA fluctuations. Our characterization of the (tension-dependent) effect of DNA fluctuations on the effective imaging resolution is highly relevant for super-resolution imaging of DNA-protein complexes that are manipulated by techniques that do not have obvious force-measuring capabilities (i.e., techniques involving flowstretched DNA, DNA curtains or DNA confined in nanochannels). Furthermore, optical tweezers are uniquely complemented by STED to enable nanoscale visualization of the force-dependent behavior of DNA-protein interactions. Access to the force dependence of kinetics and motility thus allows the unraveling of the mechanochemistry of DNA-protein interactions at the nanometer length scale and under biologically relevant conditions. We anticipate that our combined force-super-resolution approach can be readily extended to other $1 \mathrm{D}$ systems such as (trains of) molecular motors on the cytoskeleton as well as super-resolution imaging on optically manipulated cells or cellular components. Moreover, experimental access to real-time information on kinetics and motility of DNA-bound proteins at biologically relevant protein concentrations is essential for linking idealized in vitro experiments with the in vivo situation, in which DNA is densely coated with proteins.

\section{METHODS}

Methods and any associated references are available in the online version of the paper.

Note: Any Supplementary Information and Source Data files are available in the online version of the paper.

\section{ACKNOWLEDGMENTS}

We thank A.S. Biebricher, A. Candelli and S. Berning for helpful discussions and advice; J. Dikic, E. Kroezinga, T. Hoekstra and S.E.D. Haene for biochemical support; and P. Noordeloos for technical support. This work is part of the research program of the Foundation for Fundamental Research on Matter (FOM) (E.J.G.P. and G.J.L.W.), which is part of the Netherlands Organisation for Scientific Research (NWO). We acknowledge support by NWO VENI (I.H.), VICI (E.J.G.P. and G.J.L.W.) and ECHO grants (G.J.L.W.) as well as a European Research Council (ERC) starting grant (G.J.L.W.).

\section{AUTHOR CONTRIBUTIONS}

G.J.L.W. conceived the research and the multimodal approach to study mitochondrial transcription. I.H. conceived and designed the instrument and research. I.H. and G.S. built the instrument, wrote software, conceived and performed the experiments and analyzed all data. O.D.B. developed software. G.F. provided labeled TFAM and advised on experiments. W.W. and C.M. provided labeled restriction enzymes. S.W.H. advised on STED implementation and supplied phase plates. E.J.G.P. and G.J.L.W. advised on instrument design, experiments and analysis. I.H., G.S., S.W.H., E.J.G.P. and G.J.L.W. wrote the paper.

\section{COMPETING FINANCIAL INTERESTS}

The authors declare no competing financial interests.

Reprints and permissions information is available online at http://www.nature. com/reprints/index.html.

1. Cornish, P.V. \& Ha, T. A survey of single-molecule techniques in chemical biology. ACS Chem. Biol. 2, 53-61 (2007).

2. Moerner, W.E. New directions in single-molecule imaging and analysis. Proc. Natl. Acad. Sci. USA 104, 12596-12602 (2007).

3. Neuman, K.C. \& Nagy, A. Single-molecule force spectroscopy: optical tweezers, magnetic tweezers and atomic force microscopy. Nat. Methods 5, 491-505 (2008).

4. Joo, C., Balci, H., Ishitsuka, Y., Buranachai, C. \& Ha, T. Advances in single-molecule fluorescence methods for molecular biology. Annu. Rev. Biochem. 77, 51-76 (2008).

5. Candelli, A., Wuite, G.J.L. \& Peterman, E.J.G. Combining optical trapping, fluorescence microscopy and micro-fluidics for single molecule studies of DNA-protein interactions. Phys. Chem. Chem. Phys. 13, 7263-7272 (2011).

6. Moffitt, J.R., Chemla, Y.R., Smith, S.B. \& Bustamante, C. Recent advances in optical tweezers. Annu. Rev. Biochem. 77, 205-228 (2008).

7. Harada, Y. et al. Single-molecule imaging of RNA polymerase-DNA interactions in real time. Biophys. J. 76, 709-715 (1999).

8. van Mameren, J. et al. Counting RAD51 proteins disassembling from nucleoprotein filaments under tension. Nature 457, 745-748 (2009).

9. Lang, M.J., Fordyce, P.M., Engh, A.M., Neuman, K.C. \& Block, S.M. Simultaneous, coincident optical trapping and single-molecule fluorescence. Nat. Methods 1, 133-139 (2004).

10. Hohng, S. et al. Fluorescence-force spectroscopy maps two-dimensional reaction landscape of the Holliday junction. Science 318, 279-283 (2007).

11. Comstock, M.J., Ha, T. \& Chemla, Y.R. Ultrahigh-resolution optical trap with single-fluorophore sensitivity. Nat. Methods 8, 335-340 (2011).

12. Hell, S.W. Microscopy and its focal switch. Nat. Methods 6, 24-32 (2009).

13. Klar, T.A., Jakobs, S., Dyba, M., Egner, A. \& Hell, S.W. Fluorescence microscopy with diffraction resolution barrier broken by stimulated emission. Proc. Natl. Acad. Sci. USA 97, 8206-8210 (2000). 
14. Donnert, G. et al. Macromolecular-scale resolution in biological fluorescence microscopy. Proc. Natl. Acad. Sci. USA 103, 11440-11445 (2006).

15. Moneron, G. et al. Fast STED microscopy with continuous wave fiber lasers. Opt. Express 18, 1302-1309 (2010).

16. Kasper, R. et al. Single-molecule STED microscopy with photostable organic fluorophores. Small 6, 1379-1384 (2010).

17. Noom, M.C., van den Broek, B., van Mameren, J. \& Wuite, G.J.L. Visualizing single DNA-bound proteins using DNA as a scanning probe. Nat. Methods 4, 1031-1036 (2007).

18. Gittes, F. \& Schmidt, C.F. Interference model for back-focal-plane displacement detection in optical tweezers. Opt. Lett. 23, 7-9 (1998).

19. Moffitt, J.R., Chemla, Y.R., Izhaky, D. \& Bustamante, C. Differential detection of dual traps improves the spatial resolution of optical tweezers. Proc. Natl. Acad. Sci. USA 103, 9006-9011 (2006).

20. Klar, T.A., Engel, E. \& Hell, S. Breaking Abbe's diffraction resolution limit in fluorescence microscopy with stimulated emission depletion beams of various shapes. Phys. Rev. E 64, 066613 (2001).

21. van Dijk, M.A., Kapitein, L.C., van Mameren, J., Schmidt, C.F. \& Peterman, E.J.G. Combining optical trapping and single-molecule fluorescence spectroscopy: enhanced photobleaching of fluorophores. J. Phys. Chem. B 108, 6479-6484 (2004).

22. Gross, P., Farge, G., Peterman, E.J.G. \& Wuite, G.J.L. Combining optical tweezers, single-molecule fluorescence microscopy, and microfluidics for studies of DNA-protein interactions. Methods Enzymol. 475, 427-453 (2010).
23. Willig, K.I., Rizzoli, S.0., Westphal, V., Jahn, R. \& Hell, S.W. STED microscopy reveals that synaptotagmin remains clustered after synaptic vesicle exocytosis. Nature 440, 935-939 (2006).

24. Sánchez, E.J., Novotny, L., Holtom, G.R. \& Xie, X.S. Room-temperature fluorescence imaging and spectroscopy of single molecules by two-photon excitation. J. Phys. Chem. A 101, 7019-7023 (1997).

25. Thompson, R.E., Larson, D.R. \& Webb, W.W. Precise nanometer localization analysis for individual fluorescent probes. Biophys. J. 82, 2775-2783 (2002).

26. Persson, F. et al. Fluorescence nanoscopy of single DNA molecules by using stimulated emission depletion (STED). Angew. Chem. Int. Ed. Engl. 50, 5581-5583 (2011).

27. Ngo, H.B., Kaiser, J.T. \& Chan, D.C. The mitochondrial transcription and packaging factor Tfam imposes a U-turn on mitochondrial DNA. Nat. Struct. Mol. Biol. 18, 1290-1296 (2011).

28. Rubio-Cosials, A. et al. Human mitochondrial transcription factor A induces a U-turn structure in the light strand promoter. Nat. Struct. Mol. Biol. 18, 1281-1289 (2011).

29. Farge, G. et al. Protein sliding and DNA denaturation are essential for DNA organization by human mitochondrial transcription factor A. Nat. Commun. 3, 1013 (2012).

30. Patterson, G., Davidson, M., Manley, S. \& Lippincott-Schwartz, J. Superresolution imaging using single-molecule localization. Annu. Rev. Phys. Chem. 61, 345-367 (2010). 


\section{ONLINE METHODS}

DNA and proteins. DNA-dumbbell constructs were created from bacteriophage $\lambda$ DNA, biotinylated as described previously ${ }^{22}$. Streptavidin-coated microspheres (3.2- $\mu$ m diameter, Spherotech) were coupled to the $\lambda$ DNA in situ ${ }^{22}$.

The restriction enzymes were labeled as described previously ${ }^{31}$. DNA-protein complexes were obtained by preincubating $10 \mathrm{nM}$ BsoBI-Atto $647 \mathrm{~N}$ or EcoRV-Atto $647 \mathrm{~N}$ with biotinylated $\lambda$ DNA in a buffer containing $0.5 \mathrm{mM}$ EDTA, $10 \mathrm{mM} \mathrm{CaCl}_{2}, 20 \mathrm{mM}$ Tris at $\mathrm{pH} 7.6$ and $100 \mathrm{mM} \mathrm{NaCl}$. A ROXS buffer containing $2 \mathrm{mM}$ Trolox, $1 \mathrm{mM}$ methyl viologen and, for enzymatic oxygen removal, $0.43 \mathrm{mg} / \mathrm{ml}$ glucose oxidase, $72 \mu \mathrm{g} / \mathrm{ml}$ catalase and $4.5 \mathrm{mg} / \mathrm{ml} \mathrm{D}$-glucose was used to study bleaching.

TFAM was expressed, purified and fluorescently labeled with Atto $647 \mathrm{~N}$ dye as described previously ${ }^{29}$. The labeling ratio was approximately one fluorophore per two TFAM molecules. Realtime binding was studied in buffer consisting of $10 \mathrm{mM}$ Tris $\mathrm{HCl}$, pH 7.6, 25 mM NaCl, 5 mM DTT and 50 nM TFAM-Atto 647N.

Finally, Sytox Blue, Sytox Orange and Sytox Red were obtained from Invitrogen. For high coverage, 1-10 nM Sytox Blue and Sytox Orange were used, whereas individual dyes were imaged in presence of trace levels of Sytox Orange ( $100 \mathrm{pM})$. Sytox Red was imaged at $\sim 10 \mathrm{nM}$.

Microscope and microfluidics. The experiments were performed on a custom-built inverted microscope based on a water-immersion objective (CFI Plan Apo IR 60X WI, Nikon, NA 1.27) placed on a vertical stage MVN80 (Newport Corporation) using a custom-built adaptor. A five-channel laminar flow cell (Micronit Microfluidics BV) was mounted on an automated $x y$ stage (MS-2000, Applied Scientific Instrumentation), which allowed rapid, in situ construction and characterization of dumbbell constructs (typically, a construct was created in less than $1 \mathrm{~min}$ ) and facilitated swift and complete transfer of the tethered DNA between different flow channels (allowing force spectroscopy and visualization experiments to be performed on $>20$ DNA molecules per hour) ${ }^{17}$. A condenser top lens (P 1.40 OIL S1 11551004, Leica) was placed on top of the flow cell. The flow cell and microspheres were illuminated by an $875-\mathrm{nm}$ LED (LED-1115-ELC-875-19-5, IMM Photonics) and imaged in transmission onto a CMOS camera (DCC1545M, Thorlabs).

Optical trapping. Optical trapping was performed using a 10-W CW fiber laser (YLR-10-LP, $1064 \mathrm{~nm}$, IPG Photonics) with a coupled optical isolator. The laser beam was expanded using lenses with focal lengths of $75 \mathrm{~mm}$ and $150 \mathrm{~mm}$. Here, the $75-\mathrm{mm}$ lens was placed on an automated linear stage (AG-LS25, Newport) to modify the collimation for aligning the optically stretched DNA with the focal plane of the confocal imaging system. Two polarizing beam-splitter cubes (10BC16PC.9, Newport) were used to split the 1,064-nm laser into two independently steerable optical traps and recombine these. One coarse-positioning piezo stepper mirror (AG-M100N, Newport) and one accurate piezo mirror (Nano-MTA2X Aluminum, Mad City Labs) were used for beam-steering the two traps. Two 300-mm lenses coupled the trapping beams into the objective. The displacements of the trapped microspheres from the center of the trap were measured and converted into a force signal. Force measurements were performed by back-focal-plane interferometry ${ }^{18}$ of the condenser lens using two position-sensitive detectors (DL100-7PCBA3, Pacific Silicon Sensor) after separation of the two polarized beams using a polarizing beam-splitter cube. Two dichroic mirrors (950DCSP, Chroma) separated the trapping light from the LED illumination before and after the flow cell. We used approximately $1 \mathrm{~W}$ per trap, which, for $3.2-\mu \mathrm{m}$ microspheres, yields a trap stiffness of roughly $0.5 \mathrm{pN} / \mathrm{nm}$ and allows forces up to $500 \mathrm{pN}$ to be applied.

Confocal and STED fluorescence microscopy. A single, pulsed laser system (ALP-745-710-SC, $20 \mathrm{MHz}, 100$-ps pulses, Fianium) was used for fluorescence excitation and STED. This turnkey system simplifies the implementation of STED and provides flexibility in excitation wavelength. Moreover, this system allows dual-color STED (STED wavelengths $711 \mathrm{~nm}$ and $745 \mathrm{~nm}$ compatible with, for example, Atto 590 and Atto 647N) as detailed in ref. 32. In this study we have used only the 745-nm beam for single-color STED. We selected three excitation bands (centered at $467 \mathrm{~nm}, 543 \mathrm{~nm}$ and $640 \mathrm{~nm}$, compatible with a range of conventional fluorescent dyes) from a supercontinuum spectrum. After we polarized (Glan-Thompson prism PGT 1.08.05, Bernhard Halle Nachfl.) and filtered the supercontinuum spectrum using an AOTF (AOTFncVIS-TN, AA Opto-Electronic), the three beams were separated and filtered using appropriate dichroic mirrors (F43-088 and F43-093, AHF Analysentechnik) and filters (F94-640, F94-543 and F34-467, AHF). The three excitation beams and the STED beam were coupled into single-mode fibers (PMC-640 and PMC-460, Schafter \& Kirchoff) using laser beam couplers (60SMS-1-4-M15-26 and 60SMS-1-4-M15-37, Schafter \& Kirchoff) and were subsequently collimated again (collimator 60FC-L-4-M20L-02, Schafter \& Kirchoff or $f=20 \mathrm{~mm}$ achromats G052006000, Qioptiq) to yield beams of 3.6-mm diameter. Fine adjustment of the polarization angle of the 745-nm beam before fiber coupling was done using an achromatic $\lambda / 2$ retarder (RAC 4.2.10 l, Bernhard Halle). The four beams were combined using dichroic mirrors (F48-533, F33-632 and F73-726, AHF). Beam scanning using a fast tip-tilt piezo mirror (S-334.1SD, Physik Instrumente, scan rate up to $200 \mathrm{~Hz}$ ) was followed by 1:3 beam expansion and combination with the trapping laser using a dichroic mirror (F43-800, AHF). After beam expansion, the excitation and STED beams (10.8-mm diameter) overfilled the back aperture of the objective (8.5-mm diameter). All (dichroic) mirrors that encountered the STED beam were at least $6 \mathrm{~mm}$ thick and had flatness lower than $\lambda / 10$. The STED beam was centered on a custom-made binary phase plate consisting of a flat glass window that featured a $\mathrm{MgF}_{2}$ layer (approximately $1 \mu \mathrm{m}$ thick) deposited onto a section of the phase plate in order to provide a $180^{\circ}$ phase step to half of the STED beam ${ }^{20}$. In the sample, the resulting focal-intensity distribution ideally exhibits a line of zero intensity at the center of the focus such that saturated stimulated emission occurs in only the high-intensity periphery of the STED beam ${ }^{13}$. This $1 \mathrm{D}-\mathrm{STED}$ scheme can be readily adapted to $2 \mathrm{D} \mathrm{STED}{ }^{23}$ by, for example, replacing the binary phase plate with a helical phase plate and inserting a $\lambda / 4$ retarder between the phase plate and objective to establish circular polarization. Further modification including a second phase plate ${ }^{13}$ can also enable 3D STED. For confocal microscopy, the excitation beams can be circularly polarized using a $\lambda / 4$ retarder (RAC 4.4.10, Bernhard Halle). For confocal detection, the emitted fluorescence was descanned, separated from the excitation by dichroic mirrors (F48-640, F33-554, and F38-484, AHF) and filtered using appropriate emission filters (F42-652\&F47-686, F47-586, and F37-510, AHF); photons were counted using fiber-coupled APDs (APDs SPCM-AQRH-14-FC, 
fibers SPCM-QC9, PerkinElmer). The multimode fibers served as confocal pinholes ( $100-\mu \mathrm{m}$ diameter) that provide background rejection, thus increasing the signal-to-background ratio such that single fluorophores could still be resolved on the DNA even when high concentrations of labeled proteins were present in solution. Because the confocal pinhole size was large ( 1.75 Airy disks), the expected confocal imaging resolution was set by the focal-intensity distribution of the excitation beam. To block the STED beam from the detection paths, a multiphoton emission filter (F75-750, AHF) was used.

Hardware control and data acquisition. For force detection, we sampled the output voltages of the PSDs at $50 \mathrm{kHz}$ using a data acquisition card (NI PCI-4472B, National Instruments). Photon counting, beam steering and digital $\mathrm{I} / \mathrm{O}$ were performed using a multifunction card (NI PCIe-6323, National Instruments). All optical trapping, force detection and confocal fluorescence and STED hardware were controlled using custom software written in LabVIEW 2010 (National Instruments); and standard calibration, dumbbell construction, mechanical characterization and visualization procedures were largely automated. Through its clean, user-friendly interface, which can be controlled with a gaming joystick (Logitech), the LabVIEW software allows for automation of many of the complex experimental procedures. A hardware abstraction layer and a plug-in system enable the same software to run on a range of instruments in our lab.

Optical tweezers resolution. To calculate the force noise, $\sqrt{ }\left(\left\langle\delta F^{2}\right\rangle\right)$, we used the expressions derived previously in ref. 19 for the signalto-noise ratio, $\mathrm{SNR}=\langle\Delta x\rangle / \sqrt{ }\left(\left\langle\delta x^{2}\right\rangle\right)=\langle\Delta F\rangle / \sqrt{ }\left(\left\langle\delta F^{2}\right\rangle\right)$ (we used equation (3) in ref. 19 for force detection on a single bead and equation (8) in ref. 19 for the case of differential force detection). Here $\langle\Delta F\rangle$ represents a change in force due to a small change in DNA contour length, $\Delta L$, such that $\langle\Delta F\rangle=\Delta L \times k_{\text {trap }} \times k_{\mathrm{DNA}} /\left(k_{\text {trap }}+\right.$ $\left.2 k_{\mathrm{DNA}}\right)$, where $k_{\mathrm{DNA}}$ is the force-dependent stiffness of the DNA, and we assume that the two traps have equal trap stiffness, $k_{\text {trap. }}$.

Focal-intensity distribution imaging. To facilitate straightforward alignment and optimization, we used a photomultiplier tube (PMT) to directly image the focal-intensity distribution of the excitation and STED beams. A 50:50 pellicle beam splitter (BP145B1, Thorlabs) was placed in the beam path, and light, scattered by Au microspheres scanned through the focus using a piezo stage (NanoLP200, Mad City Labs), was detected using a PMT (MD 963 CPM DC, PerkinElmer). Here, 80-nm Au nanoparticles (EM.GC80/4, British Biocell International) were first mounted in a $2 \%(\mathrm{w} / \mathrm{w})$ agarose gel to avoid imaging near to the glass-liquid interface where reflections swamp the signal from the Au nanoparticles.

Longitudinal DNA fluctuations. The amplitude of the longitudinal DNA fluctuations was calculated assuming equipartition of energy as $\left\langle\sigma_{\mathrm{DNA}}{ }^{2}\right\rangle=k_{\mathrm{B}} T / K_{\|}(x)$. Here

$$
K_{\|}(x)=\left(\frac{x}{L \times k_{\mathrm{DNA}}}+\frac{1}{k_{\mathrm{trap}}}\right)^{-1}+\left(\frac{L-x}{L \times k_{\mathrm{DNA}}}+\frac{1}{k_{\text {trap }}}\right)^{-1}
$$

represents the longitudinal stiffness (i.e., in the direction parallel to the DNA axis) experienced at a point $x$ along a DNA molecule that is stretched to end-to-end length $L$. The extendible wormlike-chain model was used to approximate the tension-dependent spring constant of a section of the DNA, $k_{\mathrm{DNA}}=\partial F / \partial L$. For analysis of experimental data on BsoBI, we averaged the amplitude of the longitudinal fluctuations over the restriction sites of BsoBI on $\lambda$ DNA.

Spatial imaging resolution. The spatial resolution of STED is given by

$$
\mathrm{FWHM}_{\mathrm{STED}}=\frac{\lambda}{2 \times \mathrm{NA} \sqrt{1+\frac{P_{\mathrm{STED}}}{P_{\mathrm{S}}}}}
$$

where $\lambda$ is the excitation wavelength and $P_{S}$ is the STED power that corresponds to the saturation intensity of the fluorophore at the applied STED wavelength ${ }^{12}$. Longitudinal DNA fluctuations blur the image of DNA-bound proteins such that $\mathrm{FWHM}_{\text {meas }}=$ $\sqrt{ }\left(\mathrm{FWHM}_{\mathrm{STED}}{ }^{2}+\mathrm{FWHM}_{\mathrm{DNA}}{ }^{2}\right)$. Figure $3 \mathrm{~g}$ shows a fit of $\mathrm{FWHM}_{\text {meas }}$ to experimental data, with $P_{\mathrm{S}}$ used as a fit parameter and assuming FWHM $\mathrm{DNA}_{\mathrm{DNA}}=2 \sqrt{ }(2 \ln (2)) \sigma_{\mathrm{DNA}} \approx 31 \mathrm{~nm}$ at $4-\mathrm{pN}$ tension. Supplementary Figure 2 highlights the relative contribution of longitudinal DNA fluctuations for the fit of $\mathrm{FWHM}_{\text {meas }}$.

Localization precision. The ideal localization precision is defined as $\left\langle(\Delta x)^{2}\right\rangle=s^{2} / N+a^{2} /(12 N)+4 \sqrt{ }(\pi) s^{3} b^{2} /\left(a \times N^{2}\right)$, where the three right-hand side terms represent the mean-squared position fluctuations due to photon-counting noise, pixelation noise and background noise, and $s, a, b$, and $N$ are the s.d. of the effective focal-intensity distribution, the pixel size, the background noise and the number of photons, respectively ${ }^{25}$. To calculate the ideal localization precision for Figure 4d (solid curve), we estimated $s$ using

$$
s=\frac{\text { FWHM }_{\mathrm{STED}}}{2 \sqrt{2 \ln (2)}}=\frac{\lambda}{4 \sqrt{2 \ln (2)} \mathrm{NA} \sqrt{1+\frac{P_{\mathrm{STED}}}{P_{\mathrm{S}}}}}
$$

with $P_{\mathrm{S}}$ as determined from the fit of Figure 3g. We estimated $N$, the number of photons collected per line scan, by fitting a simple model to the experimentally determined number of photons, $N=A \times s /\left(1+B \times P_{\text {STED }}\right)$. Here, $A$ and $B$ are fit parameters that characterize, respectively, the dependence of the number of collected photons on the width of the intensity distribution and the reduction of maximum photon-emission rate due to a nonzero intensity in the minimum of the STED stripe (Supplementary Fig. 5). To determine the optimal ideal localization precision, we set $B$ to 0 (Fig. 4d, dashed curve). The experimental localization precision also includes DNA fluctuations, $\sigma_{\mathrm{DNA}}$, and additional noise, $\sigma_{\text {add }}$, associated with, for instance, drift or nonideal lineto-line-repeatability of beam scanning, such that $\left\langle(\Delta x)^{2}\right\rangle_{\text {meas }}=$ $\left\langle(\Delta x)^{2}\right\rangle+\sigma_{\mathrm{DNA}}^{2} / N+\sigma_{\text {add }}{ }^{2}$. Using $\sigma_{\mathrm{DNA}}=13 \mathrm{~nm}$ (calculated for 4-pN tension), a fit of $\sigma_{\text {add }}$ to our data in Figure 4d yields $\sigma_{\text {add }}=3.6 \mathrm{~nm}$ (Supplementary Fig. 6).

1D-diffusion simulation. We simulated diffusion trajectories by successively adding displacements $\Delta x$, drawn randomly from a Gaussian distribution with $\langle\Delta x\rangle=0$ and $\left\langle\Delta x^{2}\right\rangle=2 D_{\text {input }} \Delta t$, using a small time step $\Delta t$ of $0.4 \mathrm{~ms}$ and a diffusion constant $D_{\text {input }}$. These trajectories were binned to match the line-scan rate of our TFAM data, and white noise was added with a s.d. of 
$12 \mathrm{~nm}$ to account for the localization precision. For a given $D_{\text {input }}$, MSD analysis was performed on 100,000 trajectories, each 150 bins long $(\sim 8 \mathrm{~s})$, to approximate the distribution of measured diffusion constants.
31. Bonnet, I. et al. Sliding and jumping of single EcoRV restriction enzymes on non-cognate DNA. Nucleic Acids Res. 36, 4118-4127 (2008).

32. Bückers, J., Wildanger, D., Vicidomini, G., Kastrup, L. \& Hell, S.W. Simultaneous multi-lifetime multi-color STED imaging for colocalization analyses. Opt. Express 19, 3130-3143 (2011). 